\title{
Numerals and neural reuse
}

\section{Max Jones ${ }^{1}$}

Received: 1 February 2018 / Accepted: 27 August 2018 / Published online: 5 September 2018 (c) The Author(s) 2019, corrected publication February 2019

\begin{abstract}
Menary (in: Metzinger T, Windt JM (eds) OpenMIND, MIND Group, Frankfurt am Main, 2015) has argued that the development of our capacities for mathematical cognition can be explained in terms of enculturation. Our ancient systems for perceptually estimating numerical quantities are augmented and transformed by interacting with a culturally-enriched environment that provides scaffolds for the acquisition of cognitive practices, leading to the development of a discrete number system for representing number precisely. Numerals and the practices associated with numeral systems play a significant role in this process. However, the details of the relationship between the ancient number system and the discrete number system remain unclear. This lack of clarity is exacerbated by the problem of symbolic estrangement and the fact that unique features of how numeral systems represent require our ancient number system to play a dual role. These issues highlight that Dehaene's (in: Dehaene S, Duhamel J-R, Hauser MS, Rizolatti G (eds) From monkey brain to human brain, MIT Press, Cambridge, pp 133-157, 2005) neuronal recycling hypothesis may be insufficient to explain the neural mechanisms underlying the process of enculturation. In order to explain mathematical enculturation, and enculturation more generally, it may be necessary to adopt Anderson's (Behav Brain Sci 33(4):245-266, 2010; After phrenology: neural reuse and the interactive brain, MIT Press, Cambridge, 2014) theory of neural reuse.
\end{abstract}

Keywords Mathematical cognition · Numerical cognition · Enculturation · Neuronal recycling $\cdot$ Neural reuse $\cdot$ Cognitive integration $\cdot$ Affordances $\cdot$ Philosophy of psychology $\cdot$ Philosophy of neuroscience

Max Jones

M.D.L.Jones@leeds.ac.uk

1 University of Leeds, LG 13, Baines Wing, Leeds LS2 9JT, UK 


\section{Introduction}

Take a glimpse at the scene in front of you. Assuming that your environment isn't too cluttered, you'll immediately get a rough sense of the number of objects in your vicinity, without having to count them. 'We have a basic analogical and non-linguistic capacity to recognise number and quantity' (Menary 2015, p. 11). This capacity for rapidly apprehending the number of entities in a collection is not unique to humans and is shared by a surprisingly large range of species (De Cruz 2006), so is thought to be evolutionarily ancient. However, it is also limited by the fact that it is inherently approximate, becoming increasingly inaccurate as the size of the target collection increases (Dehaene et al. 1998). As such, the system that supports this capacity is often referred to as the approximate number system (ANS).

It is clear that the ANS cannot, on its own, explain our capacity for even basic numerical cognition, which is characteristically precise and rigorous. The question then arises as to how we are able to develop a system for precisely representing number as discrete, so that we can carry out the kind of regimented operations that are characteristic of even the most basic arithmetic. How do we develop the discrete number system (DNS) from the basis of our approximate number system (ANS)? ${ }^{1}$

Menary (2015) argues that, to answer this question, we need to look beyond what's going on in the head and pay attention to the rich cultural inheritance of cognitive technologies and practices that suffuse the environment that we develop in. The case of the development of the DNS from the ANS provides a textbook example of the process of enculturation, whereby interaction with a culturally-enriched environment gives rise to novel capacities as a result of learning-driven changes to neural structure (Menary 2015; Menary and Gillett 2016). Learning to interact with numerals and the practices associated with them gives rise to a step-change in our capacity for numerical cognition.

The emergence of the DNS has been explained in terms of forming relations between natural ANS representations of number and acquired representations of external symbols for number (Dehaene 2005; Dehaene and Cohen 2007), allowing for precise and discrete representation of number. On this picture, there is a simple overlap between the ANS and the DNS, with a 'direct mapping of symbols to quantities' (Menary 2015 , p. 13). However, this picture may be too simple. Representations in the ANS are not fine-grained enough to allow for separate representations to be associated with, for instance, the numerals " 9000 " and "10,000", and subjects are surprisingly poor at comparing symbolic and non-symbolic magnitudes for larger numbers (Lyons et al. 2012). The symbols seem to become 'estranged' from the quantities that they represent (ibid. 635). As such, the nature of the relationship between the ANS and DNS is unclear.

Although evidence suggests that our capacity for dealing with symbolic number is, in some cases, estranged from our natural capacity for recognising quantity, the

\footnotetext{
1 The term "system" is being used quite loosely here and throughout to refer to whatever fulfils the specified functional role, in line with Menary's (2015) own use of the term. As such, it should not be taken to refer to a neural system localised in a particular region of the brain. Although a more precise notion of "system" would be preferred, it is not available in the current context, since the question of how to individuate the relevant functional units is precisely what is at issue in latter parts of the paper.
} 
two capacities cannot be wholly divorced. A distinctive feature of numeral systems is that they often use number to represent number. The number of digits in a numeral tends to be systematically related to the quantity that the numeral represents. This is in contrast to ordinary language, where, in general, the structure of a symbol is not related to its content. In engaging with numeral systems, we must somehow deal with this representational duplicity, representing both the number of digits and the number that they are being used to represent.

In order to explain this capacity, it may be necessary to look beyond the neuronal recycling hypothesis as the explanation of the process of enculturation, according to which changes to our cognitive capacities are driven by learning-driven plasticity (Dehaene 2005; Dehaene and Cohen 2007; Menary 2015; Menary and Gillett 2016). In dealing with numerals, we need to exhibit a level of rapid functional flexibility that cannot be explained by this mechanism. The process of enculturation associated with the development of the DNS through our interaction with numerals is better explained by Anderson's neural reuse theory (2010, 2014), according to which much of our sophisticated cognition results from rapid and flexible recruitment of neural coalitions built from functionally diverse subsystems. This approach to understanding the brain also supports the central idea behind enculturation, that 'neural, bodily, and environmental processes all conspire to complete cognitive tasks' (Menary 2015, p. 2). While the discussion here will focus on mathematical cognition, the success of the neural reuse approach in this domain may provide reasons for adopting it to explain enculturation across the board.

The subsequent section introduces relevant evidence about numerical cognition and sets out Menary's explanation of the development of our numerical cognition capacities in terms of enculturation. Section 3 sets out the problem of symbolic estrangement (Lyons et al. 2012), and highlights the problems that it raises for Dehaene's account of the relationship between the ANS and DNS (Dehaene 2005; Dehaene and Cohen 2007). Section 4 introduces the distinctive features of how numeral systems represent, challenging the complete estrangement of numerals and quantity. Section 5 highlights the problems that this raises for accounts of enculturation based on neuronal recycling. Sections 7 and 8 present the case for the neural reuse theory (Anderson 2010, 2014) providing a better explanation of the processes underlying the development of mathematical cognition through enculturation.

\section{Numerical enculturation}

It is now well-established that humans and a surprisingly large array of other species possess a capacity for recognising the number of entities in a collection. This capacity seems to be innate and is widely held to be supported by a neural system located in the intraparietal sulcus (IPS) (see Nieder and Dehaene 2009 for review). Although reliably accurate when dealing with collections of 1-4 entities, its accuracy decreases logarithmically as the numerical magnitude of the target collection increases (Dehaene et al. 1998). ${ }^{2}$ Given that this capacity is relatively widespread across different species,

\footnotetext{
2 The ANS is not solely responsible for basic numerical abilities. The increased reliability when dealing with 1-4 objects is the result of the object-tracking system representing each object with a single mental file
} 
with a similar behavioural profile, the system is taken to be relatively evolutionarily ancient. As such, it is often referred to as 'the approximate or ancient number system (ANS)' (Menary 2015, p. 11). ${ }^{3}$

Although it is natural to think of our capacity to deal with numbers as the product of high-level cognition, the ANS is best understood as a sensorimotor system for perceiving the number of entities in concrete collections (Jones 2016a). As Dehaene puts it,

Number appears as one of the fundamental dimensions according to which our nervous system parses the external world. Just as we cannot avoid seeing objects in colour ... and at definite locations in space ... in the same way numerical quantities are imposed on us effortlessly. (1997, p. 71)

One way of explaining this as a perceptual capacity is to appeal to the ecological approach to perception, according to which we perceive affordances for possible action (Gibson 1979). Kitcher has argued that our basic access to number involves perception of affordances for engaging in counting activities, such as arranging objects into collections or spatially ordering objects (Kitcher 1984, pp. 11-12, 107-108). This approach provides a good explanation of the perceptual function of the ANS. When we perceive the numerosity of a collection of entities, we perceive the affordance for engaging in a particular counting action with respect to the objects (Jones 2018, p. 154). Kitcher's idea that we see affordances for physically manipulating the objects into collections fails to account for all cases of apparent numerical perception, such as perceiving the quantity of sounds or of unmanipulable objects like clouds. However, this problem can be overcome by understanding the relevant affordances in terms of more fine-grained actions, for example affordances for selectively attending to each object in a collection in sequence (Ibid., 156). It is easy to see why an action-oriented perceptual capacity of this kind would be evolutionarily beneficial. It enables an organism to directly perceive opportunities for enumeration, allowing them to prepare the requisite actions. For larger collections, the kind of action may only be approximately specified, yet this need not be problematic, as the organism can still prepare to engage in roughly the right kind of enumerative action, and a more accurate apprehension of number can be gained through the actual act of counting. For smaller collections of 1-4 entities, the perception of affordances for particular enumerative actions is so reliable that the animal might not need to actually count the entities. The ecological approach is by no

\section{Footnote 2 continued}

(Carey 2009; Feigenson et al. 2004). However, the fact that the object-tracking system also plays a role need not eliminate the representational role of the ANS. When subjects are presented with collections of 1-4 objects, the ANS may represent the number of objects, while the object-tracking system drives behaviour. This is supported by the finding that, when the attentional resources that support the object-tracking system are directed elsewhere, subjects' performance on numerical estimation tasks with 1-4 objects is as would be predicted if the ANS were solely responsible (Anobile et al. 2016; Burr et al. 2010).

3 There is some controversy regarding whether the ANS really represents number (Beck 2014; Burge 2010, pp. 472-483; Carey 2009). The approximate and unreliable nature of the representations may suggest that the ANS represents numerosity or approximate cardinal values rather than number (Beck 2014; Carey 2009, p. 135). However, whether or not one takes the ANS to explicitly represent number, it clearly plays a role when we engage in more abstract numerical cognition (see below), and this role still requires explanation. In line with Menary (2015), which is the main focus of the paper, I will work on the assumption that the ANS represents number. 
means the only way of explaining the function of the ANS as a perceptual capacity, and there may be reasons to reject the characterisation of the ANS in terms of numerical perception (Paz 2018). However, given the affinity of ecological approaches with Menary's approach, with both emphasising the importance of organism-environment interactions (Menary 2006, p. 331), the ecological account of the function of the ANS will be maintained as a background assumption.

A wealth of evidence suggests that the ANS is also involved in more sophisticated mathematical cognition. As one would expect from the perspective of embodied cognition, the system responsible for numerical perception also plays a significant role in numerical cognition (Jones 2016a). For example, behavioural effects associated with the apprehension of number in concrete collections also arise on tasks involving numerals or number words (Ansari et al. 2005; Dehaene et al. 1990), early ANS performance has been found to predict later performance in tasks involving numerals (Gilmore et al. 2010; Libertus et al. 2011), presentation of numerals and concrete collections causes similar patterns of neural activation (Nieder 2005; Piazza et al. 2007), and neural systems associated with numerical perception also seem to be involved in more complex arithmetical tasks, such as addition, subtraction, and multiplication (Arsalidou and Taylor 2011).

However, our sophisticated capacity for dealing with number concepts cannot be explained solely in terms of the ANS. Our ability to think about number is characteristically rigorous and precise, whereas ANS representations are increasingly approximate once one is dealing with numerical magnitudes greater than four. The question thus arises as to how we can transcend the natural capacities of the ANS and develop a discrete number system (DNS) that is able to represent each numerical value as distinct.

Engagement with external symbolic systems, such as spoken number words or written numerals, plays a significant role in the development of the DNS (Carey 2009; Spelke and Tsivkin 2001). For example, engagement with number words and numerals allows children to come to the realisation that each distinct symbol is associated with a different numerical magnitude and that larger magnitudes correspond to words [or numerals] that come later in the count list' (Carey 2009, p. 312). The precise details of how the DNS develops are a matter of contention, however, it has been argued that it involves mapping of representations of external symbols, such as numerals or number words, onto ANS representations (Dehaene and Cohen 1995, 2007). As a result of the combination of ANS representations with representations of discrete external symbols, one ends up with DNS representations that are digital and precise (Dehaene and Cohen 2007).

Menary's theory of enculturation provides a compelling story of how this stepchange in our capacity to deal with numbers occurs (Menary 2015; Menary and Gillett 2016). According to this approach, uniquely human complex cognitive capacities, such as the capacity to solve arithmetical problems, build upon phylogenetically earlier systems that evolved for sensorimotor interaction with the environment. Development in and interaction with culturally-enriched environments causes changes to the brain as a result of learning-driven neural plasticity, which give rise to functionally novel capacities. Many advances in human cognition have happened too recently to be driven by genetically-determined changes in neural structure. However, genes are not the 
only thing that we pass on. The cognitive tools and practices that we inherit from our ancestors and other conspecifics are able to shape our mind in specific beneficial ways, as a result of our being actively taught how to engage with them. Moreover, the cognitive processes that support these novel capacities are constituted by more than just neural occurrences. The cognitive processes include external representational media and other environmental scaffolds as constituents (Menary 2007).

The development of our sophisticated numerical capacities, supported by the DNS, provides a clear-cut example of enculturation, whereby novel capacities for abstract reasoning about number are built upon the ANS; the phylogenetically ancient sensorimotor system for engaging with concrete quantities. Numeral systems are a part of the cognitive scaffolding from the environment that we develop in, transforming the problem space for dealing with number and thereby shaping the neural systems that are engaged in this activity.

Although it seems clear that the development of the DNS from basic ANS resources is a case of enculturation, the exact nature of the relationship between these two systems is still unclear. On Dehaene's approach, the development of the DNS simply involves the mapping of representations of external symbols onto ANS representations as a result of neuronal recycling (Dehaene 2005; Dehaene and Cohen 2007). Neuronal recycling is involved in two ways: first, old neural systems are repurposed to respond to numeral symbols, and, second, representations of these external symbols are mapped onto representations in the ANS. As such, there is a strong overlap between the DNS and ANS, with all numeral representations mapped onto representations in the ANS. This provides a simple and complete theory, however, this theory fails to stand up to evidence. As such, the nature of the relation between the ANS and DNS requires more clarification. In what follows, it will be argued that clarifying the nature of the relation between the ANS and DNS requires a shift in the understanding of the underlying mechanisms that support enculturation.

\section{The problem of symbolic estrangement}

Until recently, it was widely accepted that the ANS plays a significant role in all numerical cognition. Although it is necessary to posit a further, culturally-determined DNS to explain sophisticated numerical capacities, it was generally agreed that the DNS resulted from augmenting the ANS by relating each representation of a distinct external symbol for number with a distinct ANS representation (Dehaene 2005). In other words, there is 'a strong overlap between symbolic and nonsymbolic number representation systems' (Lyons et al. 2012, p. 635).

Given the aforementioned evidence that signature behavioural features of the ANS emerge in subjects presented with symbolic stimuli, the idea that there is some overlap between the ANS and DNS is uncontroversial. However, both recent evidence and simple reflection on one's experience when engaging in more sophisticated mathematical cognition suggest that the proposed overlap may be less strong than has generally been assumed. In particular, the overlap between the ANS and DNS may break down 
when dealing with larger quantities. ${ }^{4}$ In such cases, our ability to understand numerical quantity may be primarily down to our culturally influenced understanding of relationships between symbols and the practices for using them, with no significant contribution from different representations in the ANS. In other words, for larger numerical quantities, the DNS may transcend the ANS and drive our capacity for sophisticated mathematical cognition independently of ANS representations.

That this is the case can be hinted at by reflecting on one's own experience. It is without doubt 'hard to imagine what a million actually looks or feels like' (Lyons et al. 2012, p. 635). However, if we had relevant ANS representation, one would expect to get some sense of million-ness that is distinct from one's sense of thousand-ness or billion-ness, even if a rather hazy and inaccurate one. Furthermore, when presented with an image containing many thousands of objects, it is very hard to guess the number of objects in the given image. For example, while it may be relatively easy to see that there were more people at Obama's inauguration than Trump's, without any relevant contextual knowledge it is pretty hard to say how many people were at either just by looking at the images. However, despite the apparent lack of natural cognitive resources for adequately representing larger quantities, we are perfectly capable of dealing with such quantities once we have developed the DNS by learning the practices associated with dealing with numerals.

These reflections on experience are further supported by experimental evidence. Lyons and colleagues assessed subjects' ability to compare quantities expressed nonsymbolically (as arrays of dots) and symbolically (as numerals or number words) (Lyons et al. 2012), with a particular focus on larger quantities for which the ANS is known to be unreliable (10-40 objects). The important trials were those in which subjects had to make mixed-format comparisons, for example, comparing a quantity in an array of dots with a quantity expressed by a numeral. In these cases, subjects performed significantly worse than when engaging in single-format comparisons, such as comparing quantity among two dot arrays or comparing quantity between two numerals. It should also be noted that subjects performed worse in symbolic single-format trials than they did in non-symbolic single-format trials. This evidence suggests that, at least once one is dealing with larger numbers for which the ANS is less reliable, comparison between non-symbolic and symbolically expressed quantities is 'considerably more difficult than comparisons of two nonsymbolic quantities' (Lyons et al. 2012, p. 639). This suggests that there is no simple mapping between symbols and ANS representations. As one moves to larger quantities, the appreciation of numerical value seems to be governed more by an understanding of the relationships between symbols than by the relationships between symbols and natural representations of quantity. The symbols become estranged from the natural system for numerical representation. This problem of symbolic estrangement demonstrates that the relationship between

\footnotetext{
4 The question of where the precise line between "smaller" and "larger" quantities lies will not be addressed here. The evidence from Lyons et al. (2012) focuses on larger numbers in the 10s as the beginning of a disconnect between ANS and DNS representation. This disconnect may be gradual, such that, for example " 40 " isn't completely estranged from the ANS, with a corresponding representation that allows one to distinguish collections of forty objects from, for instance, collections of eighty objects. Importantly, we should expect sufficiently high numbers, such as a thousand, a million, or a billion, to be fully estranged from ANS representations, since, in these cases, the ANS may just represent something like "very large quantity" in an extremely approximate fashion, thus neglecting significant differences in quantity.
} 
the ANS and the DNS cannot be as simple as Dehaene's account suggests (Dehaene 2005; Dehaene and Cohen 2007). Since there is no simple mapping from symbolic to non-symbolic representations, the overlap between the two systems is incomplete.

The fact that the overlap between the ANS and DNS is incomplete is not entirely surprising. Given the rapidly increasing inaccuracy of the ANS as numerical magnitudes get higher, it's unlikely that there would be any distinct ANS representations for numerals to be mapped onto once one reaches numbers of a certain magnitude. Furthermore, it would be very surprising indeed if augmentation with numeral representations allowed for indefinite differentiation among ANS representations, as this would involve potentially infinite representational power in presumably finite neural systems for representing number. As such, there seems to be some point at which either ANS representations play no significant role or at which numerals for large numbers are simply mapped onto a generic imprecise ANS representation of "large numerical magnitude". "Children may start out in the acquisition of discrete number systems by a mapping to an existing approximate neural coding of quantity, but as the system matures and symbols become abstracted from the ancient system, the mature system splits into two' (Menary 2015, p. 13).

In some ways, the problem of symbolic estrangement can be seen to support Menary's account of mathematical cognition in terms of enculturation, as it shows that the DNS transcends the natural ANS capacities. Engagement with external numerals and the practices related to them does not simply enhance the capacity to discriminate among ANS representations. Instead, our culturally inherited engagement with numerals gives rise to a whole new capacity that is different in kind to the function of the ANS (Menary 2015).

While the problem of symbolic estrangement helps to differentiate Menary's account from Dehaene's and highlights a more significant role for enculturation, it also renders the partial overlap between the ANS and DNS as unclear and in need of further exploration. In particular, it is unclear how DNS representations that are too large to be mapped onto precise ANS representations are able to have any specifically numerical content, rather than simply being representations of symbols that have particular norms associated with their use. Although we may be poor at appreciating large magnitudes, such as ten million, we still appreciate that the numeral " $10,000,000$ ” represents a numerical magnitude, rather than simply being the numeral that comes after "9,999,999" and before " $10,000,001$ " in a particular sequence. In order to see how one might address issues of this kind it is important to be cautious in terms of what one means by "estrangement". By suggesting that the DNS splits in two and that some DNS representations become entirely divorced from the ANS, both Lyons et al. (2012) and Menary (2015) go too far. They do so as a result of treating numerals as arbitrary symbols, akin to words. However, this overlooks the unique and idiosyncratic features of numerals as external representations. Once these features are taken into account, it is possible to develop a more detailed account of how the ANS and DNS are related, albeit one in which the relationship between the two is more convoluted than initially assumed. 


\section{The special case of numeral representations}

To fully appreciate the nature of the overlap between the ANS and the DNS, it is helpful to consider the idiosyncratic features of how numerals represent. Numerals differ from words and letters in various significant ways. The most important of these differences is that numerals are usually, to some extent, iconic, in the sense that most numeral systems utilise the number of digits that constitute a numeral as a means for representing number. ${ }^{5}$ One numerical quantity is used for representing another numerical quantity.

The earliest known external representations of number (Cain 2006), tally systems, are fully iconic, in that the number of dashes in a given tally corresponds to the number of entities represented. Turning to later numeral systems, such as Roman numerals, this iconicity is preserved in the first few numerals but the simple relationship between the number of digits in a numeral and the number represented breaks down to some extent. For example, the numeral "XVIII" contains more digits than the numeral "XX". As one turns to currently dominant place-value systems, such as the Arabic numeral system, the relationship between number of digits and number represented is somewhat recovered, in that numerals with more digits always represent higher numbers.

Henceforth, the focus will be on place-value systems that display this structural feature that distinguishes numerals from written words. One reason for this focus is that the majority of psychological studies have been conducted on subjects that have been educated to use place-values systems. As such, most of the data to be explained is within this context. In studies on subjects that haven't been educated in placevalue systems, significant differences in numerical cognition abilities have been found (Butterworth et al. 2008; Gordon 2004; Pica et al. 2004). This makes sense in relation to the theory of enculturation, which would predict that the nature of the external symbols in one's environment should affect the nature of one's cognitive processes. Thus, to understand modern enculturated mathematical cognition, it is important to see what is distinctive about currently prevalent numeral systems.

As Wiese (2003) points out, only tallies and the first few numerals of later numeral systems, such as Roman numerals, fully qualify as icons. However, the lack of full iconicity in place-value systems should not be seen as obscuring the clear differences between Arabic numerals and words or letters. In the case of written language, the shapes of linguistic representations tend to be related to the sounds of spoken words that, in turn, represent all manner of entities, whereas, in the case of numerals, number is used to represent number. ${ }^{6}$ How number is used to represent number is a matter of convention, dependent on the choice of a specific base. However, importantly, the

\footnotetext{
5 This is generally true of place-value written numeral systems, which are the main focus here. However, there are notable exceptions to this general trend, such as the Oksapmin counting system, in which body parts are used to represent number (Saxe 1982).

6 The idea that the structure of written words corresponds to the sounds of spoken words only applies to alphabetic writing systems and not to logographic writing systems, such as Japanese. Furthermore, some have questioned the extent to which the main purpose of alphabetic writing systems is the representation of sounds (e.g. Kramer and McChesney 2003), suggesting that they sometimes involve some iconic content. However, despite these variations, there is still no systematic relationship between the structure of linguistic symbols and their content that is comparable to the regimented systematic relationships between numerals and the numbers that they represent.
} 
numerical properties of the external symbols are still systematically related to the numerical values that are represented, with some structural relationship between symbol and referent, whereas, for words or letters, the relationship between the structure of the external symbol and its referent is fully arbitrary and determined by convention alone.

The numerical features of numerals are not the only factor in how these external symbols represent number in a systematic way. Numerals are also spatially regimented, with the spatial position of each digit in a numeral being semantically significant. Most numeral systems represent number using collections of digits aligned horizontally in space. In place-value numeral systems spatial position is always significant for the meaning of a given numeral, with the position in the numeral pertaining to a specific order of magnitude. Unlike the case of linguistic representation, where the structure of written words bears no structural relation to the structure of that which the words represent, in the case of numerals, the numerical and spatial features of the numeral are directly related to its representational content.

The regimented numerical and spatial structure of numerals is more than just a handy representational tool, as it enables procedures, such as "long addition" or "long division", which allow us to carry out calculations that would be extremely difficult, if not impossible, with our natural cognitive capacities alone. Zhang and Norman (1995) explain the dominance of the Arabic numeral system in terms of how its spatially regimented structure makes it more suitable for carrying out calculations using relatively simple procedures. Importantly, the beneficial nature of the Arabic numeral system is explained by the ease of the cognitive processes that we are able to exploit to make calculations with them. The numeral system is tailored to be amenable to our natural cognitive capacities. This is further reflected in the introduction of partitions at the limits of the ANS's reliability. For example, we partition large numerals, such as " $10,000,000$ " into packages of at most three digits, precisely the stage when the ANS would begin to decrease in reliability. The fact that most societies use a base-10 numeral system can also be seen to result from embodied cognitive constraints, reflecting the fact that we have ten fingers to count with. The numeral systems themselves seem to be shaped by how well they exploit our natural capacities.

The fact that numerals represent in a very different way to how words or letters represent is also reflected in the way that they are processed by the brain. Both for the case of reading and for the case of writing, different parts of the brain seem to be involved in the detection and production of numerals than are involved in the detection and production of letters and words (Carreiras et al. 2015; Longcamp et al. 2014; Park et al. 2012; Shum et al. 2013). Numeral digits seem to activate right-lateralised regions of the ventral visual system, whereas letters seem to activate left-lateralised areas (Park et al. 2012), in particular in the inferior temporal gyrus (Shum et al. 2013). This is a surprising discovery, since the differences between the external symbols that we use as digits in numerals and the external symbols that we use as letters in words are arbitrary matters of convention. If our ancestors had used the symbol "e" to represent eight-ness and the symbol " 8 " to represent various phonemes, such as /e/, this swap would presumably have had little effect on our practices. However, having established certain symbols to play each representational role, our engagement in relevant cultural practices leads to different neural structures dealing with each type of symbol. 
This is important for understanding the relationship between the ANS and the DNS, as it demonstrates two ways in which enculturation plays a role in the development of our sophisticated mathematical capacities. Firstly, our engagement with numerals provides a region of the visual system with a novel function that goes beyond our evolved capacities. While numeral systems are one of the oldest cultural inheritances that we know of, their development is still too recent in evolutionary terms for their presence in the environment to have had a significant impact on our innate neural structure. As such, the presence of brain regions that are sensitive to numerals suggests that the development of these regions must be the result of enculturation. Secondly, once the ability to recognise numerals has been established, the ability to associate these external symbols with numerical values is also a novel capacity driven by enculturation. Although numerals are to some extent iconic, many aspects of numeral representation remain a matter of convention. As such, the way in which numeral representations in the visual system get related to ANS representations must also be determined by our cultural inheritance and the cultural practices for using and manipulating numerals.

The fact that numerals represent in a very different way from letters or words leads to a different problem from the problem of symbolic estrangement. Even if larger numerals become estranged from the particular numerical values that they represent, they do not become divorced from numerical content altogether. For example, in order for us to use the numeral " 1000 " to represent one-thousand-ness, we need to represent the fact that it has four digits. If Dehaene's initial account of the strong relationship between the ANS and the DNS were correct, engaging with multi-digit numerals would seemingly involve the ANS simultaneously representing multiple numerical values, one pertaining to the number of digits in the numeral and one pertaining to the numerical value that the given numeral represents. This problem is potentially even more problematic than the problem of symbolic estrangement, since it emerges much earlier, for relatively low numbers to which the problem of symbolic estrangement supposedly doesn't apply. For example, when confronted with the numeral " 11 ", the ANS presumably has to represent both two-ness and eleven-ness. ${ }^{7}$

This problem is further exacerbated by the fact that the representational content of a given numeral inscription is highly context sensitive, depending on the particular numeral system that is in operation. For example, how one interprets "II" will depend upon the context one encounters it in. In the following sequence of tally marks or Roman numerals, "I, II, III", the "II" can be simply mapped onto the ANS representation of twoness. However, in other sequences, such as with the Arabic numerals "I0, II, I2" or the binary numerals "I, I0, II, I00", the same symbol "II" will trigger both an ANS representation of two-ness and a representation of eleven-ness or three-ness (respectively). As such, both whether the ANS serves a dual role and the specific dual

\footnotetext{
7 It may be the case that, once people become familiar with use of numeral systems, they represent multidigit numerals, such as " 11 ", holistically, rather than as two separate digits. However, this is unlikely to be the case when people are first learning to engage with numerals. Moreover, it is unlikely that people would develop the capacity to represent each numeral holistically, since there is little benefit to developing a holistic representation of each numeral as the numerals get larger, when most numerals will only be rarely encountered. In line with the theory of enculturation, one would expect the extent to which people develop holistic representations of multi-digit numerals to depend on how often they use them in their particular practice. For example, one might expect a geometer or architect to develop holistic representations of particularly salient multi-digit numerals, such as " 90 " or " 180 ".
} 
role that the ANS plays depends on context rather than simply the shape of the numeral that one encounters.

The fact that interpretation of numerals requires this dual role of the ANS may, at least in part, explain some of the difficulties people encounter in comparing nonsymbolic and symbolic numerical representations (Lyons et al. 2012). Rather than seeing subjects' diminished performance when comparing nonsymbolic and symbolic representations as resulting from a costly process of converting from one mode of representation to another, it may simply result from the fact that the ANS has to play multiple different roles, with these different roles interfering with each other. In such cases, the ANS would need to simultaneously represent three quantities: the number of dots in the nonsymbolic representation, the number of digits in the given numeral, and the numerical quantity that the numeral represents. This may also explain subjects' inferior performance when comparing between symbolic representations, since in this scenario the ANS would also need to represent both the quantity of digits in each symbol and the quantity that each symbol represents.

This issue of representational duplicity helps to highlight the complex relationship between the natural numerical abilities provided by the ANS and the culturally acquired abilities that are accomplished by the DNS. The DNS requires multiple simultaneous representations of quantity. Whether or not the ANS is able to represent multiple quantities simultaneously or whether it must do so in sequence is a matter for empirical investigation. It could be that when engaging with, for example, the numeral "100" the ANS represents both three-ness and one-hundred-ness at the same time, or it may be that it first represents three-ness and then, as a result of some outside influence from another neural system, converts this to a representation of one-hundred-ness. The important feature for current concerns is not precisely how the ANS functions in these cases but the fact that the ANS must retain its initial function for the DNS to work.

Menary's theory of mathematical enculturation is based on the idea that our neural systems gradually change their functions as a result of learning-driven neural plasticity, in line with Dehaene's neuronal recycling hypothesis. However, the representational duplicity required for dealing with numerals suggests that the development of the DNS requires that the ANS both retain its original function and flexibly deploy this function in the service of very different forms of behaviour depending on context. Thus, it is important to look in more detail at the notion of neuronal recycling in order to see how the issue of representational duplicity challenges the idea that this is the sole mechanism behind mathematical enculturation.

\section{Neuronal recycling and mathematical enculturation}

The theory of neuronal recycling has been proposed as explaining the mechanism underlying the process of enculturation. It explains how our neural structures can change as a result of our experiences in culturally-enriched environments. The neuronal recycling approach overturns the idea that the specific function of neural systems is purely evolutionarily determined. Our brain is not a tool kit consisting of an array of modules, with each module's function having evolved to fulfil a specific cognitive problem faced by our ancestors. Learning-driven plasticity caused by interaction with 
a culturally-enriched environment, can itself give rise to distinct modules with novel specific functions. As Dehaene puts it,

By neuronal recycling, I mean the partial or total invasion of a cortical territory initially devoted to a different function, by a cultural invention. [...] Neuronal recycling is also a form of reorientation or retraining: it transforms an ancient function, one that evolved for a specific domain in our evolutionary past, into a novel function that is more useful in the present cultural context. (Dehaene 2009, p. 147)

The changes in the function of a given neural system are purportedly established through synaptogenesis, i.e., changes in synaptic connectivity within that region, as well as changes in synaptic connectivity with other regions (Menary 2014). As such, neuronal recycling through learning-driven plasticity is a relatively slow process, involving neurons growing or being pruned so as to establish new connections or erase old ones. The gradual pace of change involved in changes of synaptic connectivity may, in part, explain the need for rigorous and extensive education in order to learn culturally-inherited capacities such as reading, writing, or arithmetic.

Dehaene $(2005,2009)$ provides a detailed example of how neuronal recycling works in the development of our capacity for reading. Given that written language is a relatively recent cultural development, we cannot have evolved a natural cognitive system dedicated to reading and/or writing. However, surprisingly, the same part of the brain, in the left occipito-temporal sulcus, seems to be responsible for dealing with written language across individuals and across cultures, and so has been dubbed the "visual word form area" (Dehaene 2005). Dehaene explains this finding by suggesting that learning-driven plasticity results in the repurposing of a system that would naturally be dedicated to face- and/or object-recognition to be dedicated to the detection of written forms instead. The fact that the same part of the brain is repurposed across different individuals is explained by both the stable functional profile of the natural cognitive system and by the relative stability of written forms across various cultures. This therefore presents a clear case of one form of enculturation, where our cognitive capacities are supported by both our natural cognitive systems and our interaction with a culturally developed feature of the environment.

Dehaene suggests that neuronal recycling through learning-driven plasticity is also responsible for the development of our sophisticated arithmetical capacities (2005), and Menary concurs $(2014,2015)$. Our engagement with numerals and the practices associated with them gradually lead to changes in synaptic connectivity, with certain parts of the visual system changing to respond to numerals, and these parts of the visual system becoming connected to the ANS as the numerals become associated with numerical values. However, given the differences between written language and written numerals, there are reasons to question whether the story is quite so simple.

Before questioning this approach, however, it is important to note that there is a way in which neuronal recycling is apt for explaining some aspects of our capacity to engage with numerals. Just as there were no written linguistic inscriptions in the environment in which our brain evolved, so too there were no numeral inscriptions in that environment. Which specific symbol we use to represent, for example, eight, is largely a matter of convention, in just the same way that the letter symbols that we use 
to represent language is largely a matter of convention. Presumably our language and mathematics would be functionally very similar if things had been different and " 8 " had been used to represent a particular phoneme, while "a" had been used to represent eight-ness. The fact that neuronal recycling plays some role in the representation of numerals is reflected in the fact that particular systems develop so as to be dedicated to numeral representation across individuals (Carreiras et al. 2015; Longcamp et al. 2014; Park et al. 2012; Shum et al. 2013). However, given the seemingly conventional and arbitrary nature of the decision regarding which symbols represent phonemes and which represent numbers, the fact that the system for representing letters is dissociable from the system for representing numerals already suggests that there may be something more complicated going on with the latter.

The neuronal recycling story is particularly compelling for the case of reading, since the structure of written words is arbitrarily related to the content that they represent. As such, as long as there is some system that can be repurposed to detect written words, it doesn't matter which this system is. Reading only requires that we are able to develop some system that can recognise words and that the representations in this system can then become associated with other systems that deal with the semantic categories that the words represent. The systems for detecting letters or words can still work having lost their initial functions. However, the same cannot be said of numerals, since numerals both represent number and utilise number as a means of representation.

It can't be the case that learning-driven plasticity shapes the ANS to serve a whole new purpose, as successful interaction with numerals requires the function of the ANS to be preserved. Moreover, we sometimes still need to use the ANS for its original function in the absence of numerals, having already learned to also use it in dealing with numerals, unlike the case of reading, where the visual word form area can just get on with its new job without carrying out its previous function. We need an explanation of how, in one sense, the functional profile of the ANS can remain stable, while, in another sense, the ANS can be functionally flexible. Learning-driven plasticity cannot help in explaining this, as it involves restructuring of the brain's neural connections, which takes a relatively long time to occur. The neuronal recycling approach provides a compelling story of how neural systems can change so as to acquire new functions, losing their old functions as they do so. However, in order to explain sophisticated arithmetical cognition, we need to explain how a single system can flexibly fulfil multiple functions without losing previous functionality.

\section{Neural reuse and mathematical enculturation}

Anderson's neural reuse theory $(2010,2014)$ offers a more nuanced account to the neuronal recycling hypothesis regarding how neural systems can acquire new functional roles, which may be better suited to explaining the role of the ANS in mathematical enculturation. Unlike the neuronal recycling approach, the neural reuse approach rejects the notion that local neural systems serve unique and specific behavioural functions. Neural systems are pluripotent, in the sense that each neural system can subserve a diverse range of different behavioural functions. Neural systems also display a certain degree of redundancy, with multiple systems capable of subserving the same 
behavioural function. Importantly, the particular contribution of a given neural system to a specific behavioural function at any one time is not only specified by the activity within that system. Instead, the contribution of the system is in part determined by the wider neural coalition to which it is recruited. Behavioural functionality is determined by interactions between neural systems distributed throughout the brain rather than the activity of any one locally bounded system on its own. As a result, a single system may be involved in a diverse range of behavioural tasks that one wouldn't intuitively categorise as functionally related.

Importantly, although a distinctive feature of the neural reuse hypothesis is its emphasis on the pluripotency of neural systems, this does not mean that any neural system can subserve any function. There is functional differentiation without functional specialisation. 'Some functional aspect of local circuits is relatively fixed', with local circuits having relatively stable "low-level computational "workings" that can be put to many different higher-level cognitive uses' (Anderson 2010, p. 247). Neural circuits initially evolved to fulfil a specific sensorimotor function, and the computational properties required for fulfilling the initial function are likely to be useful for other purposes. As a result, the same system can be recruited for other higher cognitive tasks, particularly when combined into a larger neural coalition, as the functional properties that emerge out of the combination of multiple more ancient subsystems may outstrip the properties of any of the members of the coalition in isolation. Rather than the functions of neural systems combining additively, the whole is more than the sum of its parts, and the specific contribution of any one subsystem on any one occasion will depend on the larger system it is a member of. " ${ }^{8}$ 'Global function is not built from componential function, but rather the reverse' (Anderson 2014, p. 93).

Like Dehaene's and Menary's accounts, the neural reuse approach suggests that learning-driven plasticity has a significant role to play in explaining how new functional capacities can emerge. However, rather than learning-driven plasticity drastically changing the internal structure of local neural systems and thereby leading to an alteration in the systems functional properties, learning-driven plasticity may be responsible for forging connections between more spatially dispersed systems to allow them to be easily recruited into neural coalitions that are capable of fulfilling novel functions. For example, in the case of numerical cognition, repeated engagement with numerals and concrete collections may increase connectivity between disparate systems, including the systems responsible for detecting numerals, the systems responsible for processing number words, the ANS, and other systems, such as the systems involved in controlling hand-movements associated with finger-counting routines or with using abacuses.

Although learning-driven neural plasticity has a role to play in neural reuse, it is not the only mechanism involved in establishing neural coalitions. As mentioned before, neural plasticity is far too gradual a process to explain the way that systems such

\footnotetext{
8 Precisely how stable the functional profiles of neural subsystems are is a matter of contention and, in more recent work, Anderson has suggested that there may even be computational functional flexibility within single systems, beyond the functional flexibility that is engendered by these systems being recruited into larger coalitions (Anderson 2014, 2016). Some have argued that his earlier (Anderson 2010) approach is more viable (Badcock et al. 2016). For present purposes, it will be argued that either of the earlier or later versions of Anderson's approach can still add significantly to our understanding of enculturation.
} 
as the ANS are able to shift functionality so rapidly. Furthermore, the neural coalitions required for explaining any one instance of sophisticated arithmetical cognition may be too spatially disparate for their coordination to be solely explained in terms of synaptic connections. However, changes in synaptic connectivity are not the only candidate for explaining changes in neural functionality. The brain may also have extra-synaptic mechanisms for coordinating subsystems relevant for a specific task on a specific occasion. One candidate for such a mechanism is the glial system, previously thought to primarily be responsible for providing nutrition to neurons, which may also play a role in functionally significant neuromodulation, both through chemical communication between glial cells and through neuron-glia interactions via regulation of synaptic transmission (Anderson 2014, p. 78; Fields 2009). Another candidate for such a mechanism is local genetic modulation resulting in changes in global synaptic network dynamics (Anderson 2014, p. 103). The precise details of which biological mechanisms the brain might use to establish the required extra-synaptic coordination are somewhat speculative, and there are further candidates that Anderson does not consider, such as exploiting the brain's endogenous electric fields (Fröhlich and McCormick 2010; Jones 2016b). However, the important point is that synaptic connectivity is not the only mechanism that determines neural function, and there are mechanisms for altering function that can operate much faster and more flexibly than changes to synaptic connectivity through neural plasticity.

As well as altering neural function through the slow process of learning-driven neural plasticity, the brain may also be able to form effective neural coalitions on the fly, as a result of extra-synaptic neural coordination. Thus, rather than understanding the brain in terms of modules with stable though mutable functions, we should understand it in terms of 'transiently assembled local neural subsystems'(Anderson 2014, p. 94), which are assembled and then recruited into wider functional networks as and when needed. Given the pluripotency and redundancy of these subsystems, different neural coalitions may be recruited to fulfil the same behavioural function from one occasion to the next. This is a particularly useful feature, since, on a given occasion, a particular subsystem may be otherwise engaged. For example, if a particular system is able to support both finger control and numerical representation but one needs to count while playing the piano, there may be another suitable alternative neural coalition available to do the job (albeit, perhaps, one with slightly diminished performance).

The neural reuse approach offers an important addition to the explanatory arsenal of enculturation approaches to mathematical cognition. Firstly, it may offer the tools to explain the representational duplicity of the ANS. Secondly it can help to explain the fact that the IPS, where the ANS is thought to be located, plays a range of roles that are not straightforwardly associated with numerical representation. Thirdly, it can help to explain how a range of different systems come to be involved in numerical representation, with the specific systems involved varying from one occasion to the next.

With regards to representational duplicity, it is not surprising, on the neural reuse approach, that the ANS can be used to represent number in different ways in the service of different behavioural functions. When simply confronted with a collection of entities, the ANS can serve its evolutionarily ancient function of numerical perception, providing an approximation of the number of entities in the collection. However, 
when we are dealing with numerals, the ANS can be recruited as part of a wider coalition, which collectively can solve the task of representing the right number. In some circumstances, the ANS might contribute a representation of the approximate number of digits in the numeral, while in others it may contribute a representation of the number represented by the numeral. In others still, it may play multiple representational roles, either simultaneously or in sequence. Which role the ANS plays on any one occasion will be determined by the overall profile of the neural coalition that it is a part of, and which coalition it is a part of will be determined by the particular task demands, with the brain recruiting an adequate coalition on the fly. Thus, the neural reuse theory can explain both the functional stability and the functional flexibility of the ANS in a way that the neuronal recycling theory alone, with its appeal to gradual functional repurposing, cannot.

The neural reuse theory is also able to explain the fact that the IPS is involved in a wide range of different tasks other than numerical approximation. For example, the IPS seems to be involved in controlling visuo-spatial attention, controlling grasping and pointing motions, motion processing, mental imagery, and working memory, among many other things (Ansari 2008; Culham and Kanwisher 2001). The fact that this region is involved in such a diverse range of behavioural functions is precisely what the neural reuse theory would predict. The neural reuse theory rejects the idea that the brain is made up of modules with specific functions, so it would be odd if the IPS was solely responsible for dealing with number. Furthermore, as an evolutionarily ancient system, one would expect the ANS to be integrated into a wider range of potential neural coalitions, 'having been available for reuse for longer' (Anderson 2010, p. 246).

Although the various functions to which the IPS seems to contribute seem to be varied and diverse, there are interesting connections between them, which may provide clues to why it is suited to supporting enculturated mathematical cognition. The IPS seems to play a role in controlling visuo-spatial attention (Gillebert et al. 2011; Grefkes and Fink 2005), and it is clear that sequential visuo-spatial attention is a key aspect of engaging in counting activities, where one must attend to each object in a collection in sequence. As such, one hypothesis regarding the role of the IPS in perceiving the number of entities in concrete collections is that the ANS is involved in perceiving affordances for engaging in certain sequences of spatial attention relevant for counting (Jones 2018). The IPS also seems to be involved in coordinating grasping and pointing actions (Simon et al. 2002), both of which, again, have obvious links to counting activities. In line with the predictions of the neural reuse hypothesis, the IPS may have initially evolved to carry out the sensorimotor tasks of controlling attention and directing hand-movements, however the computational properties that allowed it to fulfil these tasks may also have rendered it ideal for representing number (PennerWilger and Anderson 2013). However, the system in question is able to contribute to both numerical and non-numerical tasks without any drastic changes to its underlying computational properties resulting from learning-driven plasticity.

There is also plenty of evidence to suggest that the ANS is not solely responsible for our representation of number and this also supports the neural reuse approach. As has been mentioned, one would expect the system for numeral detection to also be involved in the neural coalitions responsible for representing number. However, there is also evidence that systems that represent spatial magnitudes may also be 
involved (Walsh 2003), and this is supported by behavioural evidence for various spatial-numerical association effects (Fischer and Fias 2005). Areas that are involved in language processing are also involved in numerical tasks (Delazer et al. 2003; Ischebeck et al. 2006), which is unsurprising given the importance of learning number words and memorising spoken numerical facts, such as memorising "times tables". 9 Furthermore, given the importance of finger-counting in learning about and engaging with number, it is unsurprising that systems in the left angular gyrus that play some role in controlling finger movements also play a role in representing number (Andres et al. 2007; Penner-Wilger and Anderson 2013). The reasons why these systems may play a role in numerical representation are all somewhat intuitive. However, it is important to remember that, according to the neural reuse approach, the systems that support numerical representation are often likely to be systems whose known roles are not so intuitively related to number. Moreover, the particular systems that are recruited on any one occasion may vary depending on the particular individual's history as well as the specific demands of the given task. Yet this story is exactly what one would expect from the perspective of enculturation, according to which one's cognitive scaffolding, one's learned cognitive practices, and the resources available in the immediate environment all shape the brain and contribute to the fulfilment of complex cognitive tasks.

\section{Encultured affordances as neural recruiters}

While the neural reuse theory seems apt for explaining mathematical enculturation, the question still arises regarding how we are able to recruit the appropriate neural coalition at any one time. Using "II" to represent eleven-ness, rather than two-ness or three-ness, cannot simply be a matter of transiently associating a representation in the numeral representation system with a representation in the ANS, since the same external numeral, in different contexts, must be associated with the ANS in a different manner. There must be something that ensures that we interpret "II" as eleven when dealing with Arabic numerals, as two when dealing with Roman numerals or tallies, and as three when dealing with binary. One way of addressing this problem would be to posit a "numeral-system-representation module" in the brain. However, given the relative scarcity of having to switch between different numeral systems, it would be odd if we had a system to specifically address this function. Although it is difficult, those that are new to binary or Roman numerals are able to switch their interpretation of "II" relatively quickly upon beginning to work with the new numeral system but it seems very unlikely that they had a latent Roman numeral or binary classification system in their brain lying in wait for just such an occurrence.

A better explanation of how the relevant transient neural coalitions are assembled appeals to the affordances that are available in the environment and the particular kinds of cognitive practice that one is engaging in. An account of this kind is much

\footnotetext{
9 Interestingly, evidence suggests that, in the case of expert mathematicians (Amalric and Dehaene 2016, 2018), language centres play little role in advanced mathematical cognition. This lends further support to the theory of enculturation in combination with the neural reuse hypothesis, which would predict that different systems can be used to deal with the same type of content depending on the particular individual's history and expertise and the particular task at hand.
} 
closer to the spirit of both Menary's (2007), Menary and Gillett (2016) and Anderson's (2014) accounts. The neural coalitions that we form are not merely formed from the inside, they are in part determined by the kind of actions that we are engaged in. We develop cognitive practices that enable us to recruit the right neural coalitions for a given task as and when we need them. Furthermore, our cognitive practices themselves are shaped by how we have constructed our environment. We perceive the world by perceiving affordances for action, and some of these affordances are for engaging in cognitive actions that are guided by the learned norms for a given cognitive practice (Bruineberg et al. 2018; Rietveld and Kiverstein 2014). Our structured environment shapes the kinds of cognitive practice that we develop, while we simultaneously refine the structure of our environment so that the actions relevant to the given cognitive practice are more easily achievable and the relevant affordances for those actions are more readily perceivable. Numeral systems are a particularly clear case of this, since our practice when engaging with them is extremely tightly regimented, both in terms of the spatial structure that they conform to in our environment and in terms of the norms that we learn for interacting with them.

Our neural systems initially evolved to serve sensorimotor functions, yet these systems can be recruited to perform different novel functions when incorporated into wider neural coalitions. However, questions still remain regarding how such coalitions are shaped. What enables the appropriate coalition to be selected for a given task? Anderson argues that one aspect of this selection process is internal neural recruitment mechanisms. Yet, one need not solely look inside the brain to find mechanisms for recruiting appropriate neural coalitions. Some of the recruiting work may be done by aspects of the environment (Anderson 2014, p. 283, 313). In particular, the cognitive technologies and practices that have developed over time through the process of cultural evolution may have, in part, been shaped according to their efficacy at recruiting efficient and effective neural coalitions.

In order to understand how the environment might play a role in neural recruitment, it is first important to acknowledge the close relationship between the neural reuse approach and ecological psychology (Gibson 1979). According to the latter, our perception of the world is thoroughly action-oriented. We perceive the world by perceiving affordances, which are best understood as opportunities for action in the environment. This has implications for our understanding of the brain, since perception of affordances should lead to activation in systems responsible for initiating and controlling the relevant actions, regardless of whether the action is actually carried out (Cisek 2007; Cisek and Kalaska 2010; Pezzulo and Cisek 2016). 'Interaction with an environment offering multiple affordances causes regions of the brain to be differentially activated in accordance with their functional biases' (Anderson 2016, p. 8). The affordances of the environment play a role in the recruitment of neural coalitions.

For some more basic organisms, which affordances the environment offers are largely beyond the given organism's control. The environmental niches that they occupy offer up a limited set of possible actions. However, many organisms are able to engineer their own environments to bring new affordances into being, through the process of niche construction (Laland et al. 2000; Menary and Gillett 2016; OdlingSmee et al. 2003). For example, by building dams, beavers create new affordances for sheltering that wouldn't be there otherwise. Humans are the environmental engineers 
par excellence. We have shaped our environments through buildings, tools, and a vast range of other technologies to make a plethora of new actions possible, and corresponding new affordances perceivable. Moreover, we do not only shape our environment in order to enable new possibilities for pragmatic action; we also shape them so as to enable epistemic actions that aid our cognition (Kirsh and Maglio 1994; Menary 2007, pp. 84-85). For example, when faced with the problem of counting her sheep, a shepherd can lighten the cognitive load by using a tally system, rather than keeping the number in mind, and comparing the eventual tally to a previous one. Thus, our invention of technologies and related practices give rise to new affordances for actions that serve primarily cognitive functions. Furthermore, repeated interaction with a particular technology over time, following a particular learned practice, may allow us to internalise the process, so that we can accomplish the same task by simply imagining or simulating engaging in the relevant actions. As a result, we may even perceive affordances for mental actions that involve no overt behaviour (McClelland 2017), for example, perceiving affordances for engaging in mental arithmetic.

It has only been possible to briefly gesture towards the rich literature on the way in which we inherit an environment enriched by cultural affordances here. Much more detailed accounts have been provided elsewhere (Menary 2007; Menary and Gillett 2016), and the processes described are central to the enculturation approach. The most important feature of this account is that it can be interpreted in terms of affordances. Our cognitively-scaffolded environments and the skills that we acquire to deal with them allow us to perceive an extremely rich landscape of affordances (Rietveld and Kiverstein 2014). Furthermore, these cultural affordances can serve to recruit neural systems that are tailored to fulfilling particular higher cognitive tasks (Bruineberg et al. 2018).

Many of the choices about our numeral systems and practices that may seem somewhat arbitrary, such as segmenting large numerals into packages of three digits, using a base-10 number system, or horizontally aligning the digits that make up numerals, may in fact result from features of our embodiment and the ancient neural systems that we are endowed with. We shape our external symbol systems and practices to efficiently recruit the ancient neural systems that, when combined together, can fulfil the requisite behavioural function. By developing numeral systems, we create new affordances for manipulating numerals, i.e., for literally "symbol pushing" (Landy and Goldstone 2009). Technologies such as abacuses and rod counting matrices also give rise to further numerically-relevant affordances that enable basic sensorimotor mechanisms to be recruited into coalitions for accomplishing mathematically sophisticated tasks (De Cruz 2008, 2012).

Learning to use numerals is by no means easy, and caregivers and educators tend to start teaching children about numerals from a very early age. This is, in part, because the practices for engaging with numerals are governed by strict norms. There are strict rules for how to form a numeral from digits and for carrying out addition or subtraction tasks, as well as heuristics that need to be learned for carrying out more complex arithmetical tasks. As such, the culturally specified affordances are not just shaped by the technologies that surround us but by the cognitive norms that we have learned to follow in using them (Menary 2007, pp. 136-141). The slow process of repetitively teaching relevant practices may allow for learning-driven plasticity to 
reshape our brains, which, in turn, gives rise to new affordances. When we look at the symbols on a page we can see what we can do with them, in line with the learned norms. These new affordances then enable the brain to recruit new arrays of neural coalitions to solve a given task.

An important consequence of seeing enculturation as arising from a combination of gradual learning-driven plasticity and flexible environmentally-driven online neural recruitment is that there may be no single DNS. People with different learning environments would be likely to have different neural coalitions available for recruitment in the service of numerical representation. Moreover, the neural coalition that a particular person engages from one occasion to the next is likely to vary depending on the affordances that they perceive in their environment. For example, people that were taught mathematics using abacuses may engage their visuomotor system to a greater extent when engaging in mental arithmetic than those that learned mathematics using more linguistic stimuli (Chen et al. 2006; Tang et al. 2006). However, an unskilled abacus user can still use an abacus to carry out mathematical calculations before his/her brain has had a chance to be reshaped by relevant learning-driven plasticity, with the neural coalition that is recruited to support this activity presumably being partially determined by the actions that are afforded by the abacus, as well as relevant norms acquired through observation or testimony.

\section{Conclusion}

The case of mathematical cognition is one where Anderson's theory of neural reuse is particularly useful for explaining the process of enculturation. However, given the flexibility of our encultured interactions with the world, this approach should be adopted more generally when explaining enculturation. The full story of how the encultured mind develops so that we are able to engage in complex environment-driven cognitive practices will need to explain how learning-driven plasticity and neural recruitment interact. Neuronal recycling can explain how basic biological systems can be gradually reshaped over time and thereby acquire new functional properties, while the additional mechanisms postulated by the neural reuse approach can explain how these new functional properties can be exploited in a myriad of different ways depending on the particular neural coalition that the system that has acquired these properties is recruited into. Our culturally-enriched environment both gradually reshapes our brain and flexibly alters the things that we can do with it. In order to fully understand the way our cognition emerges from our brain interacting with such a culturally rich environment, giving rise to sophisticated abilities like understanding number and solving arithmetical problems, we need to understand both the way that the brain gradually changes and the way that it can be flexibly altered in the here and now by the cultural tools in the immediate environment.

Acknowledgements This paper is based on a presentation delivered at the symposium on Mathematical Cognition and Enculturation at the European Society for Philosophy and Psychology Conference 2016. Huge thanks to Caterina Dutilh Novaes for organising this brilliant symposium and for her useful insight into this work, as well as Richard Menary, Markus Pantsar, Jean-Charles Pelland, Regina Fabry, Alexander Gillett, Christopher Burr, Zoe Drayson, and Harry Farmer for excellent discussions throughout the event 
that shaped this work. I'd also like to thank Michael Anderson for fascinating discussions elsewhere on his theory of neural reuse. Many thanks to the anonymous reviewers for their extremely helpful comments.

Open Access This article is distributed under the terms of the Creative Commons Attribution 4.0 International License (http://creativecommons.org/licenses/by/4.0/), which permits unrestricted use, distribution, and reproduction in any medium, provided you give appropriate credit to the original author(s) and the source, provide a link to the Creative Commons license, and indicate if changes were made.

\section{References}

Amalric, M., \& Dehaene, S. (2016). Origins of the brain networks for advanced mathematics in expert mathematicians. Proceedings of the National Academy of Sciences, 113(18), 4909-4917.

Amalric, M., \& Dehaene, S. (2018). Cortical circuits for mathematical knowledge: Evidence for a major subdivision within the brain's semantic networks. Philosophical Transactions of the Royal Society B, 373(1740), 20160515.

Anderson, M. L. (2010). Neural reuse: A fundamental organizational principle of the brain. Behavioural and Brain Sciences, 33(4), 245-266.

Anderson, M. L. (2014). After phrenology: Neural reuse and the interactive brain. Cambridge, MA: MIT Press.

Anderson, M. L. (2016). Précis of after phrenology: Neural reuse and the interactive brain. Behavioral and Brain Sciences, 39, 1-45.

Andres, M., Seron, X., \& Olivier, E. (2007). Contribution of hand motor circuits to counting. Journal of Cognitive Neuroscience, 19(4), 563-576.

Anobile, G., Cicchini, G. M., \& Burr, D. C. (2016). Number as a primary perceptual attribute: A review. Perception, 45(1-2), 5-31.

Ansari, D. (2008). Effects of development and enculturation on number representation in the brain. Nature Reviews Neuroscience, 9(4), 278-291.

Ansari, D., Garcia, N., Lucas, E., Hamon, K., \& Dhital, B. (2005). Neural correlates of symbolic number processing in children and adults. NeuroReport, 16(16), 1769-1773.

Arsalidou, M., \& Taylor, M. J. (2011). Is $2+2=4$ ? Meta-analyses of brain areas needed for numbers and calculations. Neuroimage, 54(3), 2382-2393.

Badcock, P. B., Ploeger, A., \& Allen, N. B. (2016). After phrenology: Time for a paradigm shift in cognitive science. Behavioral and Brain Sciences, 39, 10-11.

Beck, J. (2014). Analogue magnitude representations: A philosophical introduction. The British Journal for the Philosophy of Science, 66(4), 829-855.

Bruineberg, J., Kiverstein, J., \& Rietveld, E. (2018). The anticipating brain is not a scientist: the free-energy principle from an ecological-enactive perspective. Synthese, 195(6), 2417-2444.

Burge, T. (2010). Origins of objectivity. Oxford: Oxford University Press.

Burr, D. C., Turi, M., \& Anobile, G. (2010). Subitizing but not estimation of numerosity requires attentional resources. Journal of Vision, 10((6), 20), 1-10.

Butterworth, B., Reeve, R., Reynolds, F., \& Lloyd, D. (2008). Numerical thought with and without words: Evidence from indigenous Australian children. Proceedings of the National Academy of Sciences, 105(35), 13179-13184.

Cain, C. (2006). Implications of the Marked Artefacts of the Middle Stone Age of Africa. Current Anthropology, 47(4), 675-681.

Carey, S. (2009). The origin of concepts. Oxford: Oxford University Press.

Carreiras, M., Monahan, P. J., Lizarazu, M., Duñabeitia, J. A., \& Molinaro, N. (2015). Numbers are not like words: Different pathways for literacy and numeracy. Neuroimage, 118, 79-89.

Chen, F., Hu, Z., Zhao, X., Wang, R., Yang, Z., Wang, X., et al. (2006). Neural correlates of serial abacus mental calculation in children: A functional MRI study. Neuroscience Letters, 403(1-2), 46-51.

Cisek, P. (2007). Cortical mechanisms of action selection: The affordance competition hypothesis. Philosophical Transactions of the Royal Society of London B: Biological Sciences, 362(1485), 1585-1599.

Cisek, P., \& Kalaska, J. F. (2010). Neural mechanisms for interacting with a world full of action choices. Annual Review of Neuroscience, 33, 269-298. 
Culham, J. C., \& Kanwisher, N. G. (2001). Neuroimaging of cognitive functions in human parietal cortex. Current Opinion in Neurobiology, 11(2), 157-163.

De Cruz, H. (2006). Towards a Darwinian approach to mathematics. Foundations of Science, 11(1-2), $157-196$.

De Cruz, H. (2008). An extended mind perspective on natural number representation. Philosophical Psychology, 21(4), 475-490.

De Cruz, H. (2012). How do spatial representations enhance cognitive numerical processing? Cognitive Processing, 13(1), 137-140.

Dehaene, S. (1997). The number sense. Oxford: Oxford University Press.

Dehaene, S. (2005). Evolution of human cortical circuits for reading and arithmetic: The "neuronal recycling" hypothesis. In S. Dehaene, J.-R. Duhamel, M. D. Hauser, \& G. Rizolatti (Eds.), From monkey brain to human brain (pp. 133-157). Cambridge, MA: MIT Press.

Dehaene, S. (2009). Reading in the brain: The new science of how we read. New York, NY: Penguin.

Dehaene, S., \& Cohen, L. (1995). Towards an anatomical and functional model of number processing. Mathematical Cognition, 1(1), 83-120.

Dehaene, S., \& Cohen, L. (2007). Cultural recycling of cortical maps. Neuron, 56(2), 384-398.

Dehaene, S., Dehaene-Lambertz, G., \& Cohen, L. (1998). Abstract representations of numbers in the animal and human brain. Trends in Neurosciences, 21(8), 355-361.

Dehaene, S., Dupoux, E., \& Mehler, J. (1990). Is numerical comparison digital? Analogical and symbolic effects in two-digit number comparison. Journal of Experimental Psychology: Human Perception and Performance, 16(3), 626.

Delazer, M., Domahs, F., Bartha, L., Brenneis, C., Lochy, A., Trieb, T., et al. (2003). Learning complex arithmetic-An fMRI study. Cognitive Brain Research, 18(1), 76-88.

Feigenson, L., Dehaene, S., \& Spelke, E. (2004). Core systems of number. Trends in Cognitive Sciences, $8(7), 307-314$.

Fields, R. D. (2009). The other brain: From dementia to schizophrenia, how new discoveries about the brain are revolutionizing medicine and science. New York, NY: Simon and Schuster.

Fischer, M. H., \& Fias, M. H. (2005). Spatial representation of numbers. In J. I. D. Campbell (Ed.), Handbook of mathematical cognition (pp. 43-54). New York, NY: Psychology Press.

Fröhlich, F., \& McCormick, D. (2010). Endogenous electric fields may guide neocortical network activity. Neuron, 67, 129-143.

Gibson, J. J. (1979). The ecological approach to visual perception. Boston, MA: Houghton Mifflin.

Gillebert, C. R., Mantini, D., Thijs, V., Sunaert, S., Dupont, P., \& Vandenberghe, R. (2011). Lesion evidence for the critical role of the intraparietal sulcus in spatial attention. Brain, 134(6), 1694-1709.

Gilmore, C., McCarthy, S., \& Spelke, E. (2010). Non-symbolic arithmetic abilities and mathematics achievement in the first year of formal schooling. Cognition, 115(3), 394-406.

Gordon, P. (2004). Numerical cognition without words: Evidence from Amazonia. Science, 306(5695), 496-499.

Grefkes, C., \& Fink, G. (2005). The functional organization of the intraparietal sulcus in humans and monkeys. Journal of Anatomy, 207(1), 3-17.

Ischebeck, A., Zamarian, L., Siedentopf, C., Koppelstätter, F., Benke, T., Felber, S., et al. (2006). How specifically do we learn? Imaging the learning of multiplication and subtraction. Neuroimage, 30(4), 1365-1375.

Jones, M. (2016a). Number concepts for the concept empiricist. Philosophical Psychology, 29(3), 334-348.

Jones, M. (2016b). Review of After phrenology: Neural reuse and the interactive brain. Philosophical Psychology, 29(7), 1080-1083.

Jones, M. (2018). Seeing numbers as affordances. In S. Bangu (Ed.), Naturalizing logico-mathematical knowledge: Approaches from philosophy, psychology and cognitive science (pp. 148-163). New York, NY: Routledge.

Kirsh, D., \& Maglio, P. (1994). On distinguishing epistemic from pragmatic action. Cognitive Science, 18(4), 513-549.

Kitcher, P. (1984). The nature of mathematical knowledge. Oxford: Oxford University Press.

Kramer, S., \& McChesney, A. (2003). Writing, notational iconicity, calculus: On writing as a cultural technique. $M L N, 118(3), 518-537$.

Laland, K. N., Odling-Smee, J., \& Feldman, M. W. (2000). Niche construction, biological evolution, and cultural change. Behavioral and Brain Sciences, 23(1), 131-146. 
Landy, D., \& Goldstone, R. L. (2009). How much of symbolic manipulation is just symbol pushing. In Proceedings of the thirty-first annual conference of the cognitive science society, Amsterdam, Netherlands, July 29-August 1 (pp. 1072-1077).

Libertus, M., Feigenson, L., \& Halberda, J. (2011). Preschool acuity of the approximate number system correlates with school math ability. Developmental Science, 14(6), 1292-1300.

Longcamp, M., Lagarrigue, A., Nazarian, B., Roth, M., Anton, J. L., Alario, F. X., et al. (2014). Functional specificity in the motor system: Evidence from coupled fMRI and kinematic recordings during letter and digit writing. Human Brain Mapping, 35(12), 6077-6087.

Lyons, I. M., Ansari, D., \& Beilock, S. L. (2012). Symbolic estrangement: Evidence against a strong association between symbols and the quantities they represent. Journal of Experimental Psychology: General, 141(4), 635-641.

McClelland, T. (2017). AI and affordances for mental action. In J. Bryson, M. De Vos, \& J. Padget J (Eds.) Proceedings of AISB Annual Convention 2017 (pp. 372-379). http://aisb2017.cs.bath.ac.uk/conferen ce-edition-proceedings.pdf.

Menary, R. (2006). Attacking the bounds of cognition. Philosophical Psychology, 19(3), 329-344.

Menary, R. (2007). Cognitive integration: Mind and cognition unbounded. Basingstoke: Palgrave Macmillan.

Menary, R. (2014). Neuronal recycling, neural plasticity and niche construction. Mind and Language, 29(3), 286-303.

Menary, R. (2015). Mathematical cognition: A case of enculturation. In T. Metzinger \& J. M. Windt (Eds.), OpenMIND. Frankfurt am Main: MIND Group.

Menary, R., \& Gillett, A. (2016). Embodying culture: Integrated cognitive systems and cultural evolution. In J. Kiverstein (Ed.), The Routledge handbook of philosophy of the social mind (pp. 72-88). New York, NY: Routledge.

Nieder, A. (2005). Counting on neurons: The neurobiology of numerical competence. Nature Reviews Neuroscience, 6(3), 177-190.

Nieder, A., \& Dehaene, S. (2009). Representation of number in the brain. Annual Review of Neuroscience, 32, 185-208.

Odling-Smee, F. J., Laland, K. N., \& Feldman, M. W. (2003). Niche construction: The neglected process in evolution. Princeton, NJ: Princeton University Press.

Park, J., Hebrank, A., Polk, T. A., \& Park, D. C. (2012). Neural dissociation of number from letter recognition and its relationship to parietal numerical processing. Journal of Cognitive Neuroscience, 24(1), 39-50.

Paz, A. W. (2018). A defense of an Amodal number system. Philosophies, 3(2), 13.

Penner-Wilger, M., \& Anderson, M. L. (2013). The relation between finger gnosis and mathematical ability: Why redeployment of neural circuits best explains the finding. Frontiers in Psychology, 4, 877.

Pezzulo, G., \& Cisek, P. (2016). Navigating the affordance landscape: Feedback control as a process model of behavior and cognition. Trends in Cognitive Sciences, 20(6), 414-424.

Piazza, M., Pinel, P., Le Bihan, D., \& Dehaene, S. (2007). A magnitude code common to numerosities and number symbols in human intraparietal cortex. Neuron, 53(2), 293-305.

Pica, P., Lemer, C., Izard, V., \& Dehaene, S. (2004). Exact and approximate arithmetic in an Amazonian indigene group. Science, 306(5695), 499-503.

Rietveld, E., \& Kiverstein, J. (2014). A rich landscape of affordances. Ecological Psychology, 26(4), $325-352$.

Saxe, G. B. (1982). Culture and the development of numerical cognition: Studies among the Oksapmin of Papua New Guinea. In C. J. Brainerd (Ed.), Children's logical and mathematical cognition (pp. 157-176). New York, NY: Springer.

Shum, J., Hermes, D., Foster, B. L., Dastjerdi, M., Rangarajan, V., Winawer, J., et al. (2013). A brain area for visual numerals. Journal of Neuroscience, 33(16), 6709-6715.

Simon, O., Mangin, J. F., Cohen, L., Le Bihan, D., \& Dehaene, S. (2002). Topographical layout of hand, eye, calculation, and language-related areas in the human parietal lobe. Neuron, 33(3), 475-487.

Spelke, E. S., \& Tsivkin, S. (2001). Language and number: A bilingual training study. Cognition, 78(1), 45-88.

Tang, Y., Zhang, W., Kewel, C., Feng, S., Ji, Y., et al. (2006). Arithmetic processing in the brain shaped by cultures. PNAS, 103(28), 10775-10780.

Walsh, V. (2003). A theory of magnitude: Common cortical metrics of time, space and quantity. Trends in Cognitive Sciences, 7(11), 483-488. 
Wiese, H. (2003). Iconic and non-iconic stages in number development: The role of language. Trends in Cognitive Sciences, 7(9), 385-390.

Zhang, J., \& Norman, D. (1995). A representational analysis of numeration systems. Cognition, 5(3), 271-295.

Publisher's Note Springer Nature remains neutral with regard to jurisdictional claims in published maps and institutional affiliations. 